\title{
Einfluß des Erstkalbealters auf Fruchtbarkeit und Leistung bei Milchkuhen
}

\begin{abstract}
Summary
Title of the paper: The influence of the age of first calving on fertility and performance in dairy cows The influence of the age of first calving (respectively heifer conception age) on later fertility was investigated according to the lactation reports of 8 German herds $(n=4125)$, one US-American $(n=300)$ herd as well as to the annual average data of 206 Israeli herds.

All the correlation coefficients were set nearly zero. The optimal sphare for the age of first calving regarding some milk performance characteristics is from $24-26$ months. This can be inferred from the variance analyses from the comparisons among the groups.

The analysis of 1032 heifer births showed a rising tendency in the frequency of dystocias in higher age of first calving.
\end{abstract}

Key words: dairy cow, age of first calving, milk yield, fertility, dystocias

\section{Zusammenfassung}

Der Einfluß des Erstkalbe- bzw. Färsenkonzeptionsalters auf die spätere Milch- und Fruchtbarkeitsleistung wurde an Hand der Laktationsabschlüsse aus acht deutschen Herden $(n=4125)$, einer US-amerikanischen Herde $(n=300)$ sowie der Jahresdurchschnittsdaten von 206 israelischen Herden untersucht. Die Korrelationskoeffizienten bewegten sich nahe Null, die Varianzanalysen der Gruppenvergleiche lassen auf einen Optimalbereich für das Erstkalbealter von 24 bis 26 Monaten bezïglich einiger Milchleistungsmerkmale schließen. Die Auswertung von 1032 Färsengeburten aus drei Milchviehherden ergab für die Schwergeburtenhäufigkeit eine mit fortschreitendem Erstkalbealter steigende Tendenz.

Schlilsselwörtex: Milchrind, Erstkalbealter, Milchleistung, Fruchtbarkeit, Schwergeburten

\section{Einleitung}

Die Strategien in der Jungrinderaufzucht und das angestrebte Erstkalbealter (EKA) sind regional und waren in der Entwicklung der Rinderzucht sehr unterschiedlich. Tabelle 1 stellt diese zwischen 13 und 21 Monaten schwankenden Empfehlungen für den Zeitpunkt der ersten Besamung dar. Die Strategie zur Aufzuchtdauer muß folgende Zusammenhänge berücksichtigen:

- Reproduktionsrate und Aufzuchtdauer bestimmen den Umfang des Färsenbestandes;

- Reproduktionsrate und Erstkalbealter beeinflussen den Zuchtfortschritt;

- die Kuhleistung je Zeiteinheit hängt von Milchleistungshöhe und Aufzuchtdauer ab;

- die Aufzuchtqualităt determiniert Milch- und Fruchtbarkeitsleistungen;

- die Schwergeburtenrate ist neben anderen Faktoren auch eine Funktion des EKA. 
Tabelle 1

Literaturangaben zum empfohlenen Erstbelegungsalter (EBA) von Färsen (Recommendations on literature about the optimal age of first insemination of heifers)

\begin{tabular}{cll}
\hline EBA (Monate) & Verfasser (Land) & Jahr \\
\hline $18-21$ & A IV (DDR) & 1989 \\
$17-21$ & GOTTSCHALK et al. (D) & 1992 \\
15 & PABST (D) & 1990 \\
$16-18$ & LOTTHAMMER u. WISCHNEWSKI (D) & 1994 \\
$15-17$ & SCHWARK (DDR) & 1985 \\
$13-15$ & FRANK, ELANO (USA) & 1993 \\
& LUENING et al. (USA) & 1987 \\
$15-21$ & FEIGE (Deutschland) & 1929 \\
\hline
\end{tabular}

Auf Grundlage dieser Zusammenhänge wird durch Untersuchungen in Herden verschiedener Milcherzeugerregionen eine Empfehlung für das optimale Erstkalbealter herausgearbeitet.

\section{Literaturübersicht}

Der Eintritt der Geschlechtsreife beim Jungrind wird neben der Rassespezifität (STEWARD et al., 1980; FERRELL, 1982) von Fütterungsintensität und Wachstumsverlauf bestimmt (SCHWARK und FAHR, 1976; RIECK und ZEROBIN, 1985). Daraus ergibt sich ein Schwankungsbereich für den Beginn der Fortpflanzungsfähigkeit von 8 bis 12 Lebensmonaten (GÖTZE, 1949, zit. n. RIECK und ZEROBIN, 1985; PABST, 1990; LYHS, 1982). Das Einsetzen erster Ovulationen wie auch die Möglichkeit einer fertilen Erstbelegung hängen wesentlich stärker vom Wachstumsverlauf als vom Alter ab (HARING, 1962; SCHWARK und FAHR, 1976; SCHWARK et al., 1977; RIECK und ZEROBIN, 1985; GOTTSCHALK et al., 1992). Daneben spielen Haltungseinflüsse wie Licht, Bewegung, Klima ein Rolle (BERCHTHOLD, 1982; SCHWARK, 1985; VALENTIN, 1983).

Bei der Definition der Zuchtreife wird der notwendigen körperlichen Weiterentwicklung in Hinblick auf die Belastung einer Geburt und das spätere Milchleistungsvermögen Rechnung getragen, wobei zur ersten Zuchtbenutzung Körpermassen von 320 $400 \mathrm{~kg}$ (RIECK und ZEROBIN, 1985), 340 - $360 \mathrm{~kg}$ (FRANCK, 1993) bzw. $380 \mathrm{~kg}$ (A I 1993) gefordert werden. Verschiedene Untersuchungen, in denen eine restriktive, eine gemäße und eine intensive Fütterungsform der Jungrinder miteinander verglichen wurden, wiesen eine deutlich frühere Geschlechtsreife der intensiv aufgezogenen Tiere bei gleichem erreichten Körpergewicht nach (STAHL, 1959; SCHWARK et al., 1977; FAHR et al., 1980; GARDNER, zit. n. BERTCHTHOLD, 1982).

Auf die spätere Milch- und Inhaltsstoffleistung haben die vorpubertären Körpermassezunahmen keinen Einfluß, wenn sie zwischen 600 und $1000 \mathrm{~g} / \mathrm{Tag}$ schwanken (A II 1994). Besonders amerikanische, aber auch deutsche Empfehlungen belaufen sich auf mindestens $800 \mathrm{~g} / \mathrm{Tag}$ (A III 1993; A IV 1994), wobei die Befürchtung einer Verfettungsgefahr mit Einfluß auf Konzeption und Geburtsverlauf geäußert wird (ZEROBIN und BINDER, 1982; LANGHOLZ, 1984). Sie ist jedoch nur dann zutref- 
fend, wenn eine intensive Aufzucht nicht auch mit einer frühen Erstbelegung beantwortet wird (LEUTHOLD, 1994; PLATEN und KROCKER, 1995), zumal mit steigender Anzahl nicht genutzter Ovulationen eine sinkende Konzeptionsbereitschaft angenommen wird (STAHL, 1959; SCHWARK, 1985). In Israel liegt das EKA bereits seit Jahrzehnten bei 24 bis 26 Monaten (LEVI, 1970; KALAY, 1995; PLATEN und GROSS, 1997).

Höhere Erstlaktationsleistungen, aber niedrigere Fruchtbarkeitsleistungen ermittelten KORIATH et al. (1970) in einem Vergleich von Zwillingsschwestergruppen bei den vollwertig ernährten im Vergleich zu den verhalten ernährten Tieren.

HANSSON (zit. n. STAHL, 1959) ermittelte bei seinem klassischen Versuch mit eineiigen Zwillingspaaren für die restriktiv aufgezogenen Tiere niedrigere Milchmengenund -fettleistungen in den ersten zwei Laktationen; ab der dritten Laktation jedoch höhere Milchmengenleistungen verbunden mit einer längeren Lebensdauer gegenüber den intensiv aufgezogenen.

Sinkende Besamungsindizes ermittelte TASSELL (1967) mit steigender Fütterungsintensität, SCHWARK und LIPPMANN (1971) mit sinkendem EKA. Die ZKZ nach einem EKA von über 30 Monaten ist länger als die nach einem EKA von 25 bis 30 Monaten (RIECK und ZEROBIN, 1985). Für Färsen, die vor dem 26. Lebensmonat kalbten, ließen sich deutliche Leistungsvorteile bezüglich Milch- und Inhaltsstoffleistung gegenüber den Tieren nachweisen, deren EKA über 26 Monaten lag (PLATEN und KROCKER, 1995). Es konnte jedoch keine Abhängigkeit der folgenden Fruchtbarkeit vom EKA festgestellt werden (BERGER, 1976; PLATEN und KROCKER, 1995). Nach RIECK und ZEROBIN (1985) ist das Durchhaltevermögen während der ersten Laktation unabhängig vom EKA; bei einem EKA von über 29 Monaten ist kein Mehrertrag an Milch zu erwarten. Ein 22- bis 23-monatiges Erstkalbealter hat höhere Milchleistungen über eine längere Nutzungsdauer hinweg zur Folge (A II 1994). SCHWARK (1985) ermittelte in Bezug auf die folgende FCM-Laktationsleistung ein optimales EKA von 24,5 bis 26 Monaten und gibt eine Korrelation zwischen EKA und Laktationsleistung von - 0,2 bis - 0,4 an. Auf die Nutzungsdauer hat das Erstkalbealter keinen Einfluß (LODE, 1991; GROSS, 1997). Bei früh kalbenden Färsen hat die Körpergewichtszunahme p.p. Priorität vor der Milchleistung und Fruchtbarkeit (DUCKER et al., 1985; FERGUSON und CHALUPA, 1989). BRANDT (1985) stellte ein doppelt so hohes Energiedefizit in der Frühlaktation bei Kühen ab zweiter Laktation im Vergleich zu Erstkalbinnen fest.

\begin{tabular}{|c|c|c|c|c|c|}
\hline \multicolumn{6}{|c|}{ Zeichen- und Abkürzungserklärung } \\
\hline A & Anonym (im Literaturnachweis) & JL & Jahresleistung & PI 2:1 & Persistenzindex $2: 1$ \\
\hline BA & Besamungsaufwand & & (>305 M-Tage) & r & Korrelationskoeffizient \\
\hline EBA & Erstbelegungsalter & KR & Konzeptionsrate & RR & Reproduktionsrate \\
\hline EFQ & Biweiß-Fett-Quotient & Lakt. & Laktation & $\mathrm{RZ}$ & Rastzeit \\
\hline EKA & Erstkalbealter & ME & Milcheiweiß & Sig. & Signifikanz \\
\hline ESL & Einsatzleistung & MF & Milchfett & $\mathrm{VZ}$ & Verzögerungszeit \\
\hline FCM & Fettkorrigierte Milch & $\mathrm{MM}$ & Milchmenge & ZBZ & Zwischenbesamungszeit \\
\hline FKA & Fărsenkonzeptionsalter & M-Tage & Milchtage & ZTZ & Zwischentragezeit \\
\hline \multirow[t]{2}{*}{ IEBK } & Intervall Erstbesamung bis & NRR & Non-Return-Rate & - & Sig.niveau $(\mathrm{p}<0,05)$ \\
\hline & Konzeption (Synonym: VZ) & p.p. & post partum & $\bullet$ & Sig.niveau $(p<0,01)$ \\
\hline
\end{tabular}


Insulin fördert die Lipogenese und hemmt die Lipolyse (BLUM, 1983; EISEMANN et al., 1986; BROCKMANN und LAARVELD, 1986). Für erstlaktierende Kühe wird eine Insulinresistenz vermutet, was zu einer abweichenden Laktationsdynamik des Insulins bei Jungkühen führt. Da ein postpartal sinkender Insulinspiegel die Mobilisierung von Körpersubstanz unterstützt und bei Jungkühen im Gegensatz zu Kühen höherer Laktationen keine Senkung des Spiegels festgestellt wurde (BAUER, 1990), ist somit offenbar eine biologische Schranke vor übermäßigem Körpermasseabbau bzw. zu geringem Körpermassezuwachs in der ersten Laktation gegeben.

In früheren Jahren galt eine intensive Färsenaufzucht aus Sicht der Nährstoffökonomie als nachteilig (BERGER, 1976). Im Sinne der Stallplatzauslastung und einer schnellen Reproduktion der Herden bringt eine kurze Aufzuchtperiode Vorteile mit sich (BARZ, 1982; BERCHTHOLD, 1982; FUNK, 1993; MENZI, 1994). Die finanziellen Verluste beziffern FRANCK (1993) und LUENING et al. (1987) mit 50 US \$ je Monat, wenn das EKA über 24 Monate ansteigt. Für Deutschland werden 40 bis 50 DM angegeben (versch. Angaben, zit. n. KÜCHENMEISTER, 1993).

Die Heritabilitätsschätzungen für das Merkmal „Dystokieveranlagung“ liegen in niedrigen Bereichen und schwanken bei den Milchrassen zwischen 2 und $15 \%$ (PIRCHNER, 1981; LANGHOLZ, 1982). Mit z.T. deutlich unter 25 Monate sinkendem Erstkalbealter ist keine Häufung der Schwergeburtenfrequenz festzustellen (BERGER, 1976; BARZ, 1982). Aufgrund fortschreitenden Elastizitätsverlustes und Verfettung der Geburtswege mit zunehmendem EKA können rückläufige Schwergeburtenraten bei sinkendem Erstkalbealter ermittelt werden (DREYER, zit. n. JÄHNE, 1985; RIECK und ZEROBIN, 1985). Neben dem direkten Einfluß des EKA gelten Faktoren wie Anlage des Bullen (BERGER, 1976; THOMPSON et al., zit. n. PIRCHNER, 1981), Haltung und Fütterung (STREIT und ERNST, 1992), Körpergewicht zur Kalbung (HUTH und SMITH, 1989) sowie primäre und sekundäre Wehenschwäche, letztere in Verbindung mit Soffwechselstörungen (WEGNER, 1993), als Ursachen für Schwergeburten.

\section{Material und Methode}

Die Untersuchungen zum Einfluß des Erstkalbealters auf die Milch- und Fruchtbarkeitsleistungen wurden innerhalb einer Herde in den USA $(n=300)$ mit einer durchschnittlichen Laktationsleistung von $10.075 \mathrm{~kg}$ Milch/Kuh, anhand der Daten von 8 Herden der BRD über die Jahre 1993 bis $1996(\mathrm{n}=4125)$ mit einer durchschnittlichen Laktationsleistung von $7.500 \mathrm{~kg}$ Milch/Kuh und durch Analyse der Herdendurchschnittswerte von 206 israelischen Herden mit einer durchschnittlichen Laktationsleistung von $11.500 \mathrm{~kg} \mathrm{Milch} / \mathrm{Kuh}$ angestellt.

Die Daten entstammen den jeweiligen offiziellen Zuchtorganisationen (Holstein Frisian Association of Amerika, VIT Verden, Israel Cattle Breeder Association) und wurden mit dem Statistik-PC-Programm SPSS unter Anwendung nichtparametrischer Verfahren ausgewertet. Die Beziehung Erstkalbealter zur Schwergeburtenrate wurde unter Auswertung von 1400 Färsenkalbungen aus drei Betrieben der BRD eruiert. 


\section{Ergebnisse}

\section{Erstkalbealter und Folgeleistungen}

Tabelle 2 gibt einen Überblick über die Rangkorrelationen der Fruchtbarkeits- und Leistungsmerkmale zum Erstkalbealter. Über alle Laktationen $(n=507)$ betrachtet ergeben sich wie in den einzelnen Laktationen keine verwertbaren Zusammenhänge.

\section{Tabelle 2}

Rangkorrelationskoeffizienten (r) zwischen Erstkalbealter (EKA) und den Folgeleistungen Milch/Fruchtbarkeit (Herde USA) (Rank-correlation-coefficients ( $r$ ) between age of first calving, milk performance and fertility in the following lactations (US-dairy herd))

\begin{tabular}{lcc}
\hline & \multicolumn{2}{c}{ r zum Erstkalbealter } \\
Merkmal & 1. Lakt. & 2. Lakt. \\
\hline Milchmenge Einsatzleistung (kg) & $-0,00$ & - \\
Milchmenge 305 (kg) & 0,02 & $-0,02$ \\
Milchfett 305 (kg) & 0,04 & 0,00 \\
Milcheiweiß 305 (kg) & 0,13 & $-0,03$ \\
Milchmenge 100 (kg) & $-0,06$ & 0,09 \\
Milchmenge 200 (kg) & $-0,01$ & 0,06 \\
Persistenzindex 2:1 & 0,08 & $-0,07$ \\
Milchmenge Jahresleistung (kg) & $-0,00$ & $-0,03$ \\
Milchfett Jahresleistung (kg) & 0,03 & $-0,01$ \\
Milcheiweiß Jahresleistung (kg) & 0,02 & $-0,03$ \\
Zwischentragezeit (d) & $-0,03$ & $-0,00$ \\
\hline
\end{tabular}

Tabelle 3 zeigt die Ergebnisse des Mittelwertvergleiches verschiedener Milch- und Fruchtbarkeitsmerkmale in vier EKA-Gruppen. Es ergaben sich sowohl für die dargestellte erste Laktation als auch für die Laktationen 2 bis 5 keine statistisch abgesicherten Differenzen. Auch ein Mittelwertvergleich aller Parameter für die EKA-Gruppen $\geq$ 26,5 und $<26,5$ Monate ergab weder tendenzielle noch statistisch nachweisbare Differenzen.

Tabelle 3

Beziehungen zwischen Erstkalbealter und Folgeleistungen in der 1. Laktation (Herde USA) (Interrelation between age of first calving and performance in the first lactation (US-dairy herd))

\begin{tabular}{|c|c|c|c|c|}
\hline $\begin{array}{r}\text { EKA } \\
\text { Gruppe }\end{array}$ & $\begin{array}{c}\leq 23 \\
1\end{array}$ & $\begin{array}{c}24-26 \\
2\end{array}$ & $\begin{array}{c}27-29 \\
3\end{array}$ & $\begin{array}{c}\geq 30 \\
4\end{array}$ \\
\hline Besamungsaufwand & 2,0 & 1,9 & 2,2 & 1,6 \\
\hline Zwischentragezeit & 127 & 113 & 119 & 122 \\
\hline Milchmenge Einsatzleistung ( $\mathrm{kg}$ ) & 25 & 24 & 25 & 25 \\
\hline Milchmenge $100(\mathrm{~kg})$ & 2789 & 2700 & 2734 & 2709 \\
\hline Milchmenge $200(\mathrm{~kg})$ & 5427 & 5348 & 5341 & 5360 \\
\hline Milchmenge $305(\mathrm{~kg})$ & 7751 & 7524 & 7620 & 7680 \\
\hline Milcheiweiß $305(\mathrm{~kg})$ & 246 & 238 & 245 & 251 \\
\hline Milchfett $305(\mathrm{~kg})$ & 293 & 291 & 294 & 303 \\
\hline Persistenzindex $2: 1$ & 0,95 & 0,98 & 0,96 & 0,98 \\
\hline Eiweiß-Fett-Quotient & 0,85 & 0,82 & 0,84 & 0,84 \\
\hline
\end{tabular}

In Tabelle 4 sind die Korrelationen verschiedener Herdenleistungs- und Fruchtbarkeitsmerkmale zum Färsenkonzeptionsalter (FKA) in Auswertung der israelischen Herden dargestellt. 
PLATEN u.a.: Einfluß des Erstkalbealters auf Fruchtbarkeit und Leistung bei Milchkohen

Tabelle 4

Rangkorrelationskoeffizienten zwischen Färsenkonzeptionsalter und Leistungs- sowie Fruchtbarkeitsmerkmalen ( $n=195$ israelische Herden) (Rank-correlations-coefficients between age of first conception, milk performance and fertility ( $\mathrm{n}=195$ Israeli dairy herds))

\begin{tabular}{lc}
\hline \multicolumn{1}{c}{ Parameter } & $\begin{array}{c}\text { r mit FKA Färsen } \\
\text { (FKA=13,0-18,1) }\end{array}$ \\
\hline Reproduktionsrate Herde gesamt(\%) & $-0,10$ \\
Intervall Erstbesamung bis Konzeption Färsen (d) & $-0,16^{*}$ \\
Konzeptionsrate Färsen (\%) & $0,18^{*}$ \\
Rastzeit Erstkalbinnen (d) & $0,20^{\text {*** }}$ \\
Zwischentragezeit Erstkalbinnen (d) & 0,06 \\
Intervall Erstbesamung bis Konzeption Erstkalbinnen (d) & $-0,08$ \\
Konzeptionsrate Erstkalbinnen (\%) & 0,09 \\
Milchmenge Herde gesamt (kg) & $-0,06$ \\
Milchfett Herde gesamt (kg) & 0,00 \\
Milcheiweiß Herde gesamt (kg) & $-0,05$ \\
\hline
\end{tabular}

Tabelle 5 zeigt die Leistungsparameter in einer Gruppierung nach einem FKA von unter und über 14,5 Monaten, Tabelle 6 die Rangkorrelationen zwischen dem EKA in acht deutschen Herden und den verschiedenen Leistungs- und Fruchtbarkeitsmerkmalen. Es liegen keine nennenswerten Korrelationen vor.

Tabelle 5

Fruchtbarkeitsparameter in Herden mit einem Färsenkonzeptionsalter $\geq k 14,5$ Monate (Israelische Herden) (Parameters of fertility in herd with age of first conception $\geq K<14,5$ mounth (Israeli dairy herds))

\begin{tabular}{llccc}
\hline $\begin{array}{l}\text { Teil der } \\
\text { Herde }\end{array}$ & Parameter & FKA $\geq \mathbf{1 4 , 5}$ & Sig. & FKA $<\mathbf{1 4 , 5}$ \\
\hline \multirow{3}{*}{ Erst- } & Rastzeit & 85 & $*$ \% & 81 \\
kalbinnen & Konzeptionsrate & 41 & $*$ & 38 \\
& Intervall Erstbesamung bis Konzeption & 42 & & 47 \\
Färsen & Zwischentragezeit & 118 & & 117 \\
& Konzeptionsrate & 60 & $*$ & 57 \\
& Intervall Erstbesamung bis Konzeption & 19 & $*$ & 21 \\
\hline
\end{tabular}

Tabelle 6

Rangkorrelationskoeffizienten zwischen Erstkalbealter und Folgeleistungen in der 1. Laktation (8 Herden der BRD) (Rank-correlation-coefficients between age of first calving and performance in first lactation ( 8 German herds))

\begin{tabular}{lllllllll}
\hline Parameter & ESL & $\begin{array}{l}\text { MM } \\
\mathbf{1 0 0}\end{array}$ & $\begin{array}{l}\text { MF } \\
\mathbf{1 0 0}\end{array}$ & $\begin{array}{l}\text { ME } \\
\mathbf{1 0 0}\end{array}$ & $\begin{array}{l}\text { MM } \\
\mathbf{1 0 1 - 2 0 0}\end{array}$ & $\begin{array}{l}\text { MF } \\
\mathbf{1 0 1 - 2 0 0}\end{array}$ & $\begin{array}{l}\text { ME } \\
\mathbf{1 0 1 - 2 0 0}\end{array}$ & $\begin{array}{l}\text { MM } \\
\mathbf{3 0 5}\end{array}$ \\
\hline r zu EKA & $-0,0280$ & $-0,0175$ & 0,0371 & 0,0232 & $-0,0297$ & 0,0295 & 0,0051 & $-0,0138$ \\
(Sig.) & $(0,166)$ & $(0,388)$ & $(0,067)$ & $(0,253)$ & $(0,143)$ & $(0,145)$ & $(0,800)$ & $(0,571)$ \\
\hline Parameter & MF & ME & RZ & ZTZ & BA & IEBK & NRR 90 & ZBZ \\
& $\mathbf{3 0 5}$ & $\mathbf{3 0 5}$ & & & & & & \\
\hline r zu EKA & 0,0324 & 0,0167 & $-0,0582$ & 0,0114 & 0,0505 & 0,0487 & $-0,0408$ & 0,0496 \\
(Sig.) & $(0,182)$ & $(0,493)$ & $(0,005)$ & $(0,586)$ & $(0,016)$ & $(0,020)$ & $(0,050)$ & $(0,017)$ \\
\hline
\end{tabular}

Tabelle 7 stellt die Varianzanalyse der Milchleistungs- und Fruchtbarkeitsmerkmale nach vier EKA-Gruppen in Auswertung der acht deutschen Herden dar. Die Varianzanalysen analog zu Tabelle 7 für die Laktationen 2 bis 5 ergaben keine auswertbaren Zusammenhänge. 
Tabelle 7

Beziehungen zwischen Erstkalbealter und Folgeleistungen in der 1. Laktation (8 Herden der BRD, Varianzanalyse; a,b,c,d =signifikante Gruppendifferenz); Relationchip between age of first calving and fertility and milk performance in first lactation ( $8 \mathrm{German}$ herds, analysis of variance; ${ }^{a, b, c, d}=$ significant difference between groups)

\begin{tabular}{|c|c|c|c|c|c|}
\hline EKA (Monate) & $\begin{array}{c}\text { bis } \\
23,50 \\
\text { a }\end{array}$ & $\begin{array}{c}23,51-26,50 \\
\text { b }\end{array}$ & $\begin{array}{c}26,51-29,50 \\
\text { c } \\
\text { 1. Laktation }\end{array}$ & $\begin{array}{c}\text { ab } \\
29,51 \\
\text { d }\end{array}$ & $\underset{\text { alle }}{\bar{x}}$ \\
\hline Einsatzleistung (kg) & $21,5^{b, c, d}$ & $24,8^{2, d}$ & $24,4^{4}$ & $24,1^{2,6}$ & 24,3 \\
\hline Milchmenge $100(\mathrm{~kg})$ & $2152,4^{b, c, d}$ & $2462,9^{a, 8}$ & 2439,9 " & $2408,5^{a, b}$ & 2429,8 \\
\hline Milchmenge $101-200(\mathrm{~kg})$ & $2023,1^{b}$ & $2205,5^{\text {a.cid }}$ & $2147,0^{b}$ & $2147,6^{b}$ & 2157,5 \\
\hline Milchmenge 201-305 (kg) & 1890,8 & $1993,5^{c}$ & $1903,9^{b}$ & 1951,7 & 1938,6 \\
\hline Milchmenge $305(\mathrm{~kg})$ & $6251,8^{b}$ & $6766,2^{a, c}$ & $6579,4^{b}$ & 6619,2 & 6627,8 \\
\hline Milchfett $305(\mathrm{~kg})$ & $255,5^{b, d}$ & $275,2^{\prime \prime}$ & 271,8 & $275,1^{\prime}$ & 273,4 \\
\hline Milcheiweiß $305(\mathrm{~kg})$ & 214,8 & $229,0^{c}$ & $222,0^{b, d}$ & $226,7^{c}$ & 225,0 \\
\hline Eiweiß-Fett-Quotient & 0,8 & $0,8^{c}$ & $0,8^{\mathrm{b}}$ & 0,8 & 0,8 \\
\hline Persistenzindex 2:1 & $0,9^{b, c, d}$ & $0,9^{\mathrm{a}, \mathrm{c}}$ & $0,9^{a, b}$ & $0,9^{2}$ & 0,9 \\
\hline Rastzeit (d) & $76,1^{b, c}$ & 91,1 add & 89,2 add & $85,6^{b, c}$ & 88,2 \\
\hline Zwischentragezeit (d) & 121,5 & 120,6 & 122,6 & 122,8 & 122,2 \\
\hline Non-Return-Rate $90(\%)$ & 70 & 60 & 60 & 60 & 60 \\
\hline Zwischenbesamungszeit (d) & 20,7 & 18,0 & 19,9 & 21,1 & 19,9 \\
\hline Intervall Erstbesamung bis Konzeption (d) & 45,4 & $29,3^{d}$ & 33,2 & $36,7^{b}$ & 33,7 \\
\hline Besamungsaufwand & 1,9 & $1,6^{\mathrm{d}}$ & $1,7^{\mathrm{d}}$ & $1,8^{b, c}$ & 1,7 \\
\hline
\end{tabular}

\section{Erstkalbealter und Schwergeburtenrate}

Die Abbildung zeigt den prozentualen Schwergeburtenanteil bei Erstkalbinnen aus drei deutschen Herden. Von einem Genanteil von mindestens $85 \%$ Holstein Friesian kann ausgegangen werden. Der Anteil an Erstkalbinnen mit Schwergeburten beläuft sich auf $20,7 \%$ bei den Tieren, deren EKA $\geq 27$ Monate betrug und differierte damit signifikant $(p<0,05)$ zum Schwergeburtenanteil von $15,1 \%$ bei den Färsen, deren EKA unter 27 Monaten lag.

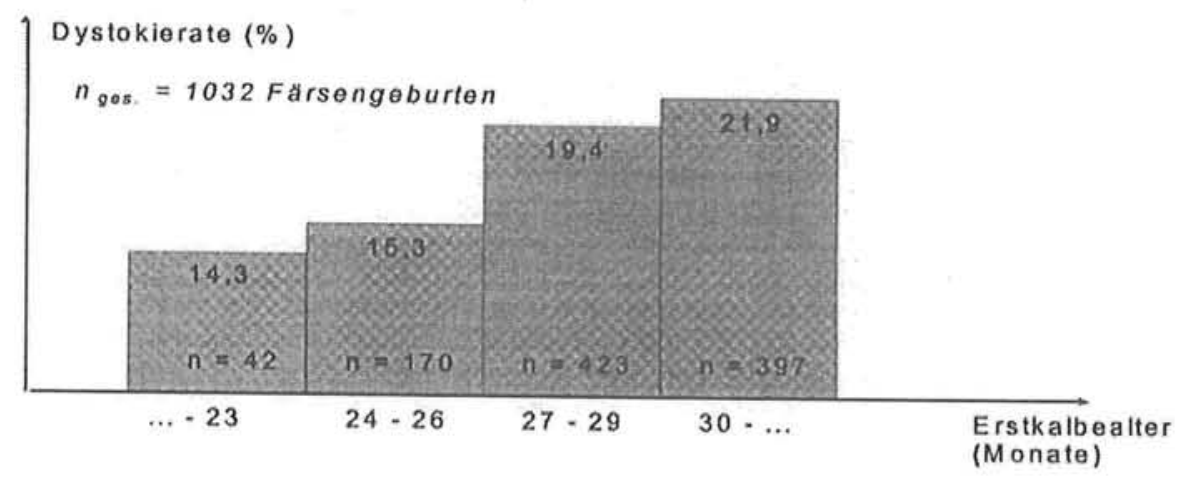

Abb.: Schwergeburtenrate nach EKA-Gruppen, Daten der Jahre 1992-1997/1995-1997 aus drei deutschen Herden (Age of first calving in classes and frequency of dystocia, calvings in 3 dairy herds in the years 1992 to 1997 and 1995 to 1997) 


\section{Diskussion}

Die von LEUTHOLD (1996) beschriebene natürliche Frühreife des Rindes, die sich im Erlangen der Fortpflanzungsfähigkeit bereits mit 8 bis 10 Monaten (PABST, 1990) bzw. 8 bis 12 Monaten (LYHS, 1982; RIECK und ZEROBIN, 1985) äußert, ist durch eine zeitige Belegung nutzbar. Schon zu früheren Zeiten war dies bekannt (FEIGE, 1929).

Die Rangkorrelationskoeffizienten zwischen FKA und verschiedenen Leistungs- und Fruchtbarkeitsmerkmalen im Rahmen der Analyse der israelischen Kibbuzherden ergaben lediglich für die Beziehung zum IEBK der Färsen eine signifikante, aber geringe Korrelation von $\mathrm{r}=-0,16^{*}$ und zur KR von $\mathrm{r}=0,18^{*}$, wenn das FKA zwischen 13 und 18 Monaten schwankt.

Darüber hinaus waren weder zu den Herdenleistungen der Färsen noch zu denen der Kühe in erster Laktation Beziehungen nachweisbar. Die Milchfettleistung war in Herden mit dem früheren FKA leicht verringert, der EFQ schwach signifikant erniedrigt in Herden mit höherem FKA. Als Ursache kann die geringere Körpermasse der Erstkalbinnen mit der Folge eines niedrigeren Fettgehaltes vermutet werden.

Die signifikant längere Rastzeit in Herden mit höherem FKA ist vermutlich auf das Management zurückzuführen: der Betriebsleiter, der seine Färsen bewußt später besamt, wird auch mentalitätsgebunden seinen Kühen die längere RZ einräumen.

Die mit drei Prozent überlegenere KR bei den Färsen bzw. Erstkalbinnen mit einem FKA über 14,5 Monaten fiel zwar gering, allerdings nicht unbedeutend aus. Darüber hinaus konnten keine Unterschiede bezüglich Milchleistung oder Fruchtbarkeit in Abhängigkeit vom FKA zwischen den Herden ausgemacht werden. Das signifikant um zwei Tage differierende IEBK liegt in beiden Fällen innerhalb des Bereiches eines Fortpflanzungszyklus.

Für die Leistungsmerkmale der US-amerikanischen Herde ergaben sich keine Zusammenhänge in den Laktationen 1 bis 5 zum EKA - wie sie etwa HANSSON (zit. n. STAHL, 1959) beschreibt. Auch die Gruppierung in EKA-Bereiche ergab keine signifikanten Leistungsunterschiede; allerdings war in der Gruppe EKA 24 - 26 Monate die ZTZ mit 113 Tagen am niedrigsten. Die Milchleistung der bis 23 Monate kalbenden Färsen war am höchsten, was Angaben nach A II (1994) nahe kommt; ihre ZTZ war allerdings die längste, was indirekt Angaben nach KORIATH et al. (1970) entspricht, und die Persistenz die niedrigste. Bei Analyse der Beziehungen zwischen Leistung und EKA in den acht deutschen Herden konnten keine Korrelationen ermittelt werden, wie sie etwa SCHWARK (1985) angibt. Bei Einteilung in vier EKA-Gruppen allerdings offerierte die Varianzanalyse für alle Laktationen die z.T. signifikant, z.T. nicht signifikant besten Leistungen und für die 1. Laktation auch die besten Fruchtbarkeitsergebnisse für die Kühe der EKA-Gruppe 23,51-26,5. Damit können Ergebnisse von SCHWARK (1985) bestätigt werden, nach denen ein 24,5 bis 26 -monatiges EKA bezüglich Milchleistung als optimal herausgestellt wurde. Die deutlich niedrigeren Milchleistungen der bis zum 23. Lebensmonat kalbenden Färsen der deutschen Betriebe stehen im Gegensatz zu den israelischen Färsen derselben EKA-Gruppe: ihre Milchleistung war die höchste. Dies ist vermutlich auf die allgemein sehr viel höhere 
Fütterungsintensität in der israelischen Zuchtpraxis im Gegensatz zur deutschen zurückzuführen.

Mit den Ergebnissen kann zunächst die amerikanische und israelische Zuchtpraxis, dargelegt von FRANCK (1993), ELANO (1993) und LUENING et al. (1987), als ökonomisch und leistungsunterstützend eingeschätzt werden, sofern sich das EKA zwischen 24 bis 26 Monaten bewegt. Eine Erstkalbung vor dem 24. Monat ist im Resultat der eigenen Untersuchungen wegen fruchtbarkeitsdepressiver Tendenzen abzulehnen, desselben eine zu späte Erstkalbung. Ebenso wie durch RIECK und ZEROBIN (1985) konnte durch die eigenen Untersuchungen keine Einflußnahme des EKA aufs Durchhaltevermögen nachgewiesen werden. Die Leistungsvorteile für früher kalbende Färsen und die relative Unabhängigkeit der folgenden Fruchtbarkeit - soweit das EKA über 23 Monaten liegt - wie sie BERGER (1976) sowie PLATEN und KROCKER (1995) feststellen, bestätigten sich. Kein durch die verschiedenen Untersuchungsbetriebe durchgehender Konsens dagegen besteht zu den nach A V (1993) geschilderten Leistungsvorteilen für Erstkalbinnen mit einem 22 bis 23-monatigen EKA.

Nach HARING (1962), SCHWARK und FAHR (1976), SCHWARK et al. (1977), RIECK und ZEROBIN (1985) sowie GOTTSCHALK et al. (1992) muß die Bedeutung des erreichten Körpergewichtes vor das Alter gesetzt werden, welches weit in den Hintergrund tritt, sofern die Aufzuchtintensität hohe Zunahmen ermöglicht. Die durch ZEROBIN und BINDER (1982) und LANGHOLZ (1984) geschilderten verfettungsbedingten Nachteile für solch intensiv ausgezogene Jungrinder, wie sie die israelischen und amerikanischen darstellten, konnten nicht erkannt werden.

Ausgehend von der Annahme, daß in den drei untersuchten Herden für die Färsen keine Bullen mit ausgewiesenem Zuchtwert für große Kälber oder Schwergeburten herangezogen wurden und in allen Fällen eine Untergrenze bezüglich des Körpergewichtes zum Zeitpunkt der Besamung realisiert wurde, ist der dritte, hauptsächliche Faktor, das Alter der Kuh bei Erstbesamung resp. Erstkalbung, unter Vernachlässigung der beiden Erstgenannten in seiner Beziehung zur Schwergeburtenrate untersucht worden. Der Verlauf der Dystokierate bei einer speziellen Gruppierung der Kühe in EKABereiche ist nahezu linear steigend. Wenn diese Steigerung der Dystokierate mit steigendem EKA auf Grund fehlender Signifikanz nicht als zwingend interpretiert werden darf, so kann doch zumindest die oftmals vorliegende Befürchtung und von ZEROBIN und BINDER (1982) sowie LANGHOLZ (1984) geschilderte Gefahr einer mit sinkendem EKA sich vollziehenden Häufung der Dystokien nicht bestätigt werden. Die Ergebnisse sprechen für ein frühes EKA. Die ermittelten Dystokieraten entwickelten sich in Abhängigkeit vom EKA ähnlich wie die nach A VI (1985) sowie DREYER, zit. n. JÄHNE (1985), lagen insgesamt aber höher, was auf die andersartigen Genotypen zurückgeführt werden könnte.

Die abnehmende Elastizität der Geburtswege scheint mit der Erhöhung des EKA um wenige Monate größere Wirkung zu zeigen als das in diesen Monaten wachsende Bekken. Offenbar ist das bovine Becken zur ersten Kalbung immer relativ zu klein, und von der Elastizität bzw. dem Verfettungsgrad der Geburtswege hängt es ab, ob dieses relativ zu kleine Becken für eine Schwergeburt verantwortlich zeichnet oder einen 
normalen Geburtsverlauf zuläßt. Es konnte kein Anhaltspunkt dafür gefunden werden, daß hohe Aufzuchtintensität zur Verfettung der Geburtswege führt, wenn diese intensive Fütterung mit einer frühen Zuchtbenutzung einher geht, wie es LEUTHOLD (1996) darstellt. Im Ergebnis der ermittelten Tendenz wird eine erhöhte Schwergeburtenfrequenz offensichtlich durch andere Faktoren, nicht jedoch durch ein sinkendes EKA bedingt.

\section{Schlußfolgerungen}

Ein frühes Erstkalbealter von 24 bis 26 Monaten hat leistungsphysiologische Vorteile gegenüber einem höheren und niedrigeren. Die natürliche Frühreife des Rindes ist somit im Sinne hoher Leistungen nutzbar. Der Anteil der Dystokien bei den Erstkalbinnen wird durch eine Vorverlegung des Erstkalbealters nicht erhöht; er sinkt jedoch deutlich bei Unterschreitung eines 27-monatigen im Vergleich zu darüber liegendem Erstkalbealter.

Eine Verlängerung der Zwischentragezeit bei Hochleistungskühen kann durch die Vorverlegung des Zeitpunktes der ersten Kalbung kompensiert werden, womit ein positiver Effekt auf die Lebensleistung gegeben ist. Die bereits in vielen Ländern erfolgreich realisierte Erstkalbung im Alter von 24 bis 26 Monaten sollte auch die Zuchtpraxis in Deutschland beeinflussen.

\section{Literatur}

ANONYM I:

Körpergewichte zur Zucht und Kalbung Empfehlungen der Coburn Comp. auf dem Maßband zur ANONYM II: Brustumfangsmessung bei Kühen, 1993

ANONYM III:

Amerikanische Zuchtpraxis, zit. n. FRANCK, RHONDA (1993)

ANONYM IV:

In: Osnabrücker Schwarzbuntzucht (1994) 3, 40-41

ANONYM V:

Untersuchungen der Ohio-State University, zit. n. FRANCK, RHONDA (1993)

ANONYM VI:

BARZ, T.: In: AID-Heft 295, 1985

Die ökonomische Wertung des Verfahrens der extremen Frühnutzung weiblicher Jungrinder. HumBAUER, J.: boldt-Universität Berlin, Diss. vet.-med., 1982

Untersuchungen zur Anwendung der invasiven Rückenfettdickenmessung beim Rind. Humboldt-Universität Berlin, Diss. vet.-med., 1990

BERCHTHOLD, M.:

In: BERCHTHOLD, M. u. E. GRUNERT: Fertilitätsstörungen beim weiblichen Rind. Parey Verlag Berlin/Hamburg, 1982

BERGER, G.:

Zum Einfluß der Stall- und Weidehaltung im Färsenalter auf die Fruchtbarkeit der Färsen sowie den Geburtsverlauf und die Fruchtbarkeit der Jungkühe in einer industriemäßigen Milchviehanlage. Mh. Vet.- Med., Jena 35 (1976), 124- 127 
BLUM, J.W.:

Endokrinologie und Tierproduktion. Schweizer Arch. Tierheilkunde. 125 (1983), 827

BRANDT, A.: Futteraufnahme und Energiebilanz zu Beginn der Laktation. Univ. Kiel., Diss. agr., 1985

BROCKMANN, R.P.; LAARVELD, B.: Hormonal regulation of metabolism in ruminants - a review. Livestock Prod. Sci., Amsterdam 14 (1986), 313-334

DUCKER, M.J.; HAGGET, R.A.; FISHER, W.J.; MORANT, S.V.; BLOOMFIELD, G.A.: Nutrition and reproductive performance of dairy cattle. 1. Anim. Prod., Edinburgh 41 (1985), 1

EISEMANN, J.H.; HAMMOMD, A.C.; BAUMANN, D.E.; REYNOLDS, P.J.; TYRRELL, H.F.; HAALAND, G.L.: Effects of bovine growth hormone administration on metabolism of growing hereford heifers. J. Nutr. 116 (1986), 2504

ELANO ANIMAL HELTH Keep heifers raising in the profit zone. Dairy Herd Management 10 (1993), 39-40

FAHR, R.D.; SCHWARK, H.-J.; JÄHNE, M.; EBERT, B.: Der Einfluß der Wachstumsintensität und der genetischen Konstruktion auf die Reproduktionsleistung wachsender Rinder. Mh. Vet. Med., Jena 35 (1980), 921-924

FEIGE, E.: Haustierkunde und Haustierzucht. Verlag Quelle u. Meyer Leipzig, 1929, 40

FERGUSON, J.D.; CHALUPA, W.: Symposium: Interactions of nutrition and reproduction. J. Dairy Sci., Champaign, Ill. 72 (1989), 746766

FERRELL, C.L.:

Effects of postweaning rate of gain on onset of puberty and productive performance of heifers of FRANCK, R: different breeds. J. Anim. Sci., Albany, N.Y. 55 (1982), 1272-1283

FRANCK, R: FUNK, D.: Who should be raising your heifers? Dairy Herd Management (1994) 3, 74

Are production and reproduction genetically linked? Holstein world, N.Y. (1993) 11, 33- 60

GOTTSCHALK, A.; ALPS, H.; ROSENBERGER, W.:

In: Praktische Rinderzucht und Rinderhaltung. Verlagsunion Agrar München, 1992

GROSS, U.:

HARING, P.:

(Hachaklait Veterinärverband Israel); pers. Mitt., 1997

Zielfragen in der Rinderzucht, I. u. II. Der Tierzllchter 21 (1962), 550- 552

HUTH, F.-W.; SMIDT, D.:

JÄHNE, M:

Genetischer Fortschritt ist nicht alles. Der Tierzilchter.(1989) 2, 434-435

Einflußfaktoren auf Milchleistung u. Milchzusammensetzung. In: SCHWARK, H.-J.: Rinderzucht. KALAY, D.: DLV Berlin. 155. ff., 1985

Pers. Mitteilung des Sci. and Prod. Manager of the Israel Cattle Breeders Assoc, 1995

KORIATH, G.; SCHÜLER, W.; GIRSCHEWSKI , H.:

Der Einfluß der Aufzuchtintensität der Färsen auf die spätere Fruchtbarkeit und Gesundheit. Mh. Vet.Med., Jena 25 (1970), 492-494

KUCCHENMEISTER, U.:

Vorstellung eines Servicesystemes zur Fruchtbarkeitsüberwachung. Vortrag auf der Tagung für Fortpflanzung landw. Nutztiere des IFN Schönow und der HU Berlin am 10.10.1993 in Bernau

LANGHOLZ, H.-J.:

Züchterische Möglichkeiten zur Verbesserung der Fruchtbarkeit beim Rind. Der Tierzüchter 11 (1982), 368-371

LANGHOLZ, P.:

Aufzuchtintensităt und Nutzungsdauer. Der Tierzilchter 36 (1984), 337- 339 
LEUTHOLD, G.:

Pers. Mitteilung, 1993, 1996

LEUTHOLD, G.:

Züchterische Beeinflußbarkeit der Fruchtbarkeit. Vortrag auf der Fruchtbarkeitstagung des IFN LEVI, U.: Schönow e.V. u. der HU Berlin am 10.10.1994 in Bernau

Aufbau und Entwicklung der schwarzbunten Milchviehpopulation in Israel. Der Tierzüchter 29 (1970), 3

LODE, E.J.:

Genetisch-züchterische Analyse zur Nutzungsdauer des Schwarzbunten Milchrindes. Humboldt-Universität Berlin, Diss. agr., 1991

LUENING, R.A.; KLEMME, M.; HOWARD, T.

Wisconsin farm enterprise budget-dairy cows and replacementes, University of Wisconsin-Extension

LYHS, L.: Public. A 2731, 1987

MENZI, B.

Physiologie der landwirtschaftlichen Nutztiere. S. Hirzel Verlag. Leipzig, 1982, 2

PABST, W.:

Cullings in dairy cows. Hoard's Dairyman v. 25.09.1994

Rinderproduktion. In: GRANZ, E., J. WEISS, W. PABST u. K.E. STRACK: Tierproduktion, 11. Aufl.

PIRCHNER, F.: P. Parey Verlag, 1990, 273-368

Genetischer Antagonismus in der Rinderzucht. Der Tierzüchter 33 (1981) 2, 48-52

PLATEN, M.; KROCKER, M.:

Leistungsvorsprung in der Färsenproduktion - Durch intensive Aufzucht zu früher Erstkalbung. Berlin. Neue Landwirtschaft 7 (1995), 63-65

PLATEN, M.; GROSS, U.:

Schattendächer und Beregner für die weltbesten Milchkühe. Zucht, Haltung, Management und Organisation der israelischen Milchviehhaltung.- Berlin, DBV, Bauernzeitung (1997)14, 38-39

RIECK, G.W.; ZEROBIN, K.:

Biologische und betriebswirtschaftliche Voraussetzungen einer ungestörten Fertilität; Genetik der Fruchtbarkeit. In: Zuchthygiene Rind, Pareys Studientexte, Berlin u. Hamburg. 18-52 u. 105-112, 1985

SCHWARK, H.-J.; FAHR, R.-D.:

Untersuchungen zum Wachstum, Eintritt der Geschlechtsreife und zu Merkmalen der Fruchtbarkeit beim Rind. 1. Mitt. Arch. Tierz., Berlin 19 (1979), 75-85

SCHWARK, H.-J.; LIPPMANN, E.:

Untersuchungen über den Einfluß eines herabgesetzten Erstkalbealters auf die Körperentwicklung und die Leistung von Färsen des Deutschen Schwarzbunten Rindes bis zum Abschluß der Erstlaktation. Arch. Tierz., Berlin 14 (1971), 163-175 u. 267-282

SCHWARK, H.-J.:

In: SCHWARK, Rinderzucht. 2. Aufl., Landwirtschaftsverlag, Berlin, 1985, 268-280 u. 211-221

SCHWARK, H.-J.; FAHR, R.-D.; BREUNUNG, R.; EBERT, S.:

Wachstum und Pubertät beim Rind unter dem Aspekt der frühzeitigen reproduktiven Nutzung. 26. Int. Fachtag. Fortpfl. Bes. Haust. Wels/Österreich, 1977

STAHL, W.:

Der Einfluß der Ernährung auf die Leistung und Körperform. Beeinflussung physiologischer Leistungen. In: Handbuch für Tierzüchter, Bd. 1, Leipzig, 1959, 393-398

STEWARD, T.S.; LONG, C.R.; CARTWRIGHT, T.S.:

Characterization of cattle of a five-breed diallel. III. Puberty in bulls of heifers. J. Animal Sci., Albany, N.Y. 50 (1980), 808- 820

STREIT, P.; ERNST, E.:

Einflüsse auf peri- und postnatale Kälberverluste unter besonderer Berücksichtigung der Haltungsbedingungen. 1.: Einflüsse auf perinatale Kälberverluste. Züchtungskunde, Stuttgart 64 (1992) 1, 35-44

TASSELL, R.:

The effects of diet on reproduction in pigs, sheeps and cattle. Brit. Vet. J. 123 (1967), 459- 463 
VALENTIN, A.:

Untersuchungen zur Wachstums- und Fruchtbarkeitsleistung von Färsen. Humboldt-Universität Berlin, Diss., 1991

WEGNER, W.:

Genetisch bedingte Geburtsstörungen. In: RICHTER, J. u. R. GÖTZE (1993): Tiergeburtshilfe. P. Parey 4. Aufl., 1993, 280-286

ZEROBIN, K.; BINDER, H.:

Biologische und wirtschaftliche Aspekte der Fruchtbarkeit beim Rind. Der Tierzilchter 34 (1982), 366368

Eingegangen: 21.04 .1999

Akzeptiert: 09.07.1999

Anschriften der Verfasser

Dr. MATTHIAS PLATEN

Thuringer Lehr-, Prüf- und Versuchsgut GmbH

Am Feldschloßchen 9

D-99439 Buttelstedt

Dr. MANFRED KROCKER, Prof. Dr. Dr. h.c. mult. ERNST LINDEMANN

Humboldt-Universität zu Berlin

Landwirtschaftlich-Gärnerische Fakultät

Institut fulr Nutztierwissenschaften

Philippstr. 13

D-10115 Berlin

Dr. URI GROSS

Israelischer Veterinärverband Hachaklait

Elisha Nr. 10

Kareit Gat

Zip-Code 82000

Israel 


\title{
Buchbesprechung
}

\author{
Damwild und Rotwild in landwirtschaftlichen Gehegen - Ein Leitfaden für Haltung, Fütterung, \\ Gesundheitsüberwachung, Verwertung und Ökonomik
}

\author{
HERMANN BOGNER (Hrsg.)
}

2. neubearbeitete Auflage, 300 Seiten, 84 Abbildungen, 63 Tabellen, Parey Buchverlag, im Blackwell Wissenschaftsverlag, Berlin/Wien, 1999, ISBN 3-8263-3287-3, 78,00 DM, 569,00 ÖS, 72,00 SFr

Die Haltung von Wildtieren in der Landwirtschaft hat in den letzten zwei Jahrzehnten in Deutschland, in europäischen und anderen Ländern einen steigenden Stellenwert erhalten. Wegbereiter für diese Entwicklung waren, vor allem in Deutschland, Wissenschaft und Forschung. Das gilt sowohl für die ehemalige DDR als auch für die BRD. Die mit diesen Arbeiten verbundene Öffentlichkeitsarbeit verschiedenster Form lieferte das Rüstzeug für die Umsetzung in die Praxis. Inzwischen werden z.B. in der Europäischen Gemeinschaft in mehr als 9600 Haltungen über 100000 Tiere des Dam- und Rotwildes gehalten. Sowohl die umweltfreundliche Haltung bei der Nutzung des natürlichen Grünlandes in extensiver oder intensiver Wirtschaftsweise als auch die Erzeugung hochwertiger Fleischqualitäten findet zunehmend größeres Verbraucherinteresse und begünstigt diese landwirtschaftliche Nischenproduktion.

Sicher ist aber auch, daß nur ausreichendes Fachwissen um die Haltung von Wildtieren in Gehegen Garant fuir den Erfolg dieser Tierhaltung ist. Es verwundert daher nicht, daß sich auf Grund des großen Interesses für den Herausgeber und Verlag nach relativ kurzer Zeit die Notwendigkeit ergab, diese neubearbeitete 2. Auflage herauszubringen. Herausgeber und Mitautoren haben bei dieser Ausgabe, soweit es erforderlich war, neueste Ergebnisse und Erfahrungen eingearbeitet, neue Gesetzesvorschriften ergänzt und im Kapitel Ernährung die Umstellung des Energiegehaltes in Mega-Joule vorgenommen. Der bewährte, übersichtliche Buchaufbau blieb unverändert. Im ersten Hauptabschnitt werden die gesetzlichen Bestimmungen der Haltung von Wildtieren in Gehegen dargelegt. Es folgen die Besprechung von Dam- und Rotwild, die Einrichtung landwirtschaftlicher Gehege sowie das Management und die Besonderheiten der Herdenhaltung von Dam- und Rotwild. Weitere Hauptabschnitte widmen sich der Ernährungsphysiologie und Fütterungspraxis, Krankheiten, Krankheitsvorbeuge und Immobilisation, Wildbretbehandlung und -verwertung. Besonders wichtig für Einsteiger ist der letzte Abschnitt zur Ökonomie der Dann- und Rotwildhaltung in Gehegen in dem u.a. die Wirtschaftlichkeit der Damund Rotwildhaltung, Aspekte des Einkommensbeitrages aus der Dam- und Rotwildhaltung in Gehegen aber auch der Wettbewerbsvergleich extensiver Tierhaltungsverfahren erörtert wird.

Jedem Hauptabschnitt ist ein weiterfuhrendes Literaturverzeichnis nachgeordnet. Ein Sachwortverzeichnis erleichtert die Arbeit mit diesem übersichtlich gegliederten und alle wichtigen Elemente der Gehegehaltung enthaltenden Buch. Gute Bilder und Abbildungen sowie zahlreiche Tabellen und Öbersichten unterstützen die verständlichen und anwendungsorientierten Texte. Als zusammengefaßter Leitfaden für die Haltung, Fütterung, Gesundheitsüberwachung, Verwertung und Ökonomik der beschriebenen Wildarten ist dieses praxisrelevante Werk eine Anleitung zum Handeln. Es ist Landwirten, die sich im Haupt- oder Nebenerwerb mit der Wildhaltung in Gehegen vertraut machen wollen, unentbehrlich. Dieses Buch ist Landwirten, vor allem Tierhaltern, -züchtern und -schützern, Studierenden der Landwirtschaft und Veterinärmedizin aber auch Wild und Jagd verbundenen Lesern zu empfehlen. 